\title{
Experiências de avaliação do setor suplementar de saúde: contribuições da integralidade
}

\author{
Evaluation experiences of the supplementary care sector: \\ contributions of integrality
}

Aluísio Gomes da SilvaJ unior ${ }^{1}$

M arcia Guimarães de M ello Alves ${ }^{1}$

M onica Tereza M achado M ascarenhas ${ }^{1}$

Valeria M arinho Nascimento Silva ${ }^{1}$

Luis Cláudio deCarvalho ${ }^{1}$

${ }^{1}$ Departamento de Planejamento em Saúde, Instituto deSaúdeda Comunidade, UFF. Rua M arquês do Paraná 303/ 3o andar/Anexo ao HUAP, Centro. 24030-210 Niteró RJ. agsilvaj@uninet.com.br
Abstract This work identifies aspects of compre hensiveness in the proposals for healthcare evaluation of the Agência Nacional de Saúde Suplementar (AN S) in Brazil, and of the National Commitee Quality Assurance (NCQA) in the U.S.A. Theinvestigation was based on a documental analysis of the proposals found in the websites of both institutions in the second semester of 2006. The data were systemized according to pre established attributes, building an analytical matrix for evaluating the following three dimensions: manage ment practices and service organization; quality of knowledge and of practices of the health workers; and quality of the control practices of the society. In the Brazilian case there was an emphasis on the first and the third dimensions. The evaluation focalized theoperators; thehealth plans were not evaluated. In the American case there was a balance between the three sets of attributes and the evaluation was focused on the health plans. Both proposals measured the satisfaction of the beneficiaries and made the results of the evaluations available in their websites. In conclusion we emphasize the importance of evaluative concepts and approaches for measuring the integrality of privatehealth plan operators in the Brazilian supplementary health sector.

Key words Performance measurement, Quality of health care, Integrality, Health services, Supplementary care
Resumo Este trabalho identifica aspectos de integralidade nas propostas de avaliação de assistência à saúde da A gência Nacional de Saúde Suplementar (ANS), no Brasil, e do N ational Commitee for Quality Assurance (NCQA), nos EstadosU nidos. A metodologia adotada foi a da análisedocumental das propostas en contradas nos sítios eletrôni cos das instituições no segundo semestre de 2006. Os dados foram sistematizados numa matriz analítica com atributos das práticas de gestão e organização de serviços; dos conhecimentos e práticas dos trabalhadores de saúde; e das práticas de controle pela sociedade. 0 s resultados apresentados destacam, no caso brasileiro, uma ênfase no primeiro e terceiro atributos, com a avaliação centrada nas operadoras - os planos de saúde não foram avaliados. No caso americano, houve um equilíbrio nos três conjuntos de atributos, sendo a avaliação direcionada aos planos de saúde. Ambas as propostas mensuraram a satisfação dos ben eficiários e divulgam os resultados das avaliações em sítios eletrônicos. Em conclusão, destaca-se a importância de concei tos eabordagens avaliativas da integralidade nas operadoras de planos privados de saúde no setor suplementar brasileiro.

Palavras-chave Avaliação de desempenho, Q ualidade da assistência à saúde, Integralidade, Serviços de saúde, Saúde suplementar 
Introdução

A integralidade como um dos princípios do Sistema Ú nico de Saúde (SUS) tem suscitado interessante debate na tentativa de transformá-la em conceito(s) operacional (ais) que sirva( $m$ ) à avaliação dos progressos assistenciais do Sistema de Saúde ${ }^{1-4}$.

Os sentidos da integralidade encontrados na literatura internacional apresentam desde atributos como acesso, capacidade dos profissionais de perceber os usuários em suas dimensões biopsicossociais, articulação da equipe multiprofissional nos serviços até oferta de serviços que compreendam desde a promoção da saúde à reabilitação de seqüelas e a possi bilidade de arti culação de serviços em níveis crescentes de sofisticação tecnológica para resolução dos problemas da população $0^{4}$.

As experiências de avaliação queusam a integralidade como eixo ainda são escassas, porém trazem à tona algumas questões para reflexão. Diferentementeda literatura internacional, o conceito de integralidadeno Brasil tem agregado significados e contextos próprios, tais como a luta pela instituição, manutenção e ampliação de direitos sociais recém-adquiridos; as enormes desigualdades socioeconômicas e culturais; a insuficiência das redes assistenciais; os modelos de gestão freqüentemente centralizados everticaise o descompasso entre as necessidades de atenção dos usuários e os serviços disponíveis4.

O Laboratório de Pesquisas sobre Práticas de Integralidade em Saúde (LAPPIS) tem sido importante ator no debate brasileiro na tentativa de ampliação dos sentidos da integralidade para buscar respostas às singularidades do nosso contexto ${ }^{5-8}$.

Ampliando os sentidos da integralidade na avaliação

Ao se assumir a integralidade como eixo prioritário de uma política de saúdea ser desenvolvida tanto no setor público quanto no setor privado de saúde, deve-se ter em mentea necessidade de dois movimentos recíprocos dos sujeitos envolvidos nesses processos organizativos. Um de les é a superação dos obstáculos existentes no âmbito do setor saúde (tais como as políticas econômicas e sociais excludentes; a grande prevalência de sofrimento difuso na população; a insuficiência derecursosfinanceiros do setor saúde e a cultura institucional normativa e vertical na instituição de políticas públicas na saúde) e para além deste. 0 outro movimento correspon- de à implantação de inovações no cotidiano dos serviços de saúde?.

Essas inovações podem ocorrer nas relações entre os níveis de gestão do SUS ou nas relações destes gestores com a sociedade. Sua construção deve ser gerada a partir de permanentes interações dos sujeitos nos serviços eentreeles, sempre pautadas por valores que busquem a emancipação, tais como: a garantia da autonomia, o exercício da solidariedade e o reconhecimento da liberdade de escolha do cuidado e da saúde que se deseja obter. Os serviços de saúde constituem espaços públicos onde se encontram "sujeitos coletivos", produtos de suas intersubjetividades, mas ainda carentes de um agir político, compartilhado e socializado ${ }^{10}$.

Assim, a integralidade deve ser tomada como uma bandeira política, indissociável da universalidade e da eqüidade (outros princípios), e ser materializada, no cotidiano dos serviços de saúde, em práticas de gestão, de cuidado e de controle pela sociedade. Estas práticas devem buscar a produção de espaços de diálogo entre os vários atores que interagem nos serviços, o desenvolvimento demelhor percepção das necessidades dos usuários e trabal hadores da saúde e a mediação e pactuação das ações articuladas de cuidado nos diferentes níveis de atenção.

O cuidado deve ser, pois, uma ação integral com significados e sentidos voltados para a compreensão de saúde como um direito do ser enão como um nível de atenção do sistema ou como um procedimento simplificado ${ }^{9}$. Essa ação integral deverá resultar de uma nova forma de relação das pessoas ${ }^{11}$ e os efeitos e repercussões das interações positivas entre os usuários, os profissionais e as instituições, e pode ser traduzida em atitudes como tratamento digno respeitoso, com qualidade, acolhimento evínculo. 0 cuidado também se traduz na escolha ética e política das tecnologias a serem utilizadas na resolução dos problemas de saúde ${ }^{12,13}$.

O controle pela sociedade precisa ser construído com mecanismos de participação social, vocalização de atores em assimetrias de poderes e pela capacidade coletiva para avaliar o desempenho das políticas públicas. Uma forma possível de operacionalizar a avaliação do cuidado foi proposta por Silva Junior e M ascarenhas ${ }^{4}$ por meio dos conceitos de acolhimento, vínculo-responsabilização e qualidade da atenção à saúde. Acolhimento incluiria as noções de acesso, referência, capacidade de escuta e percepção das demandas e seus contextos biopsicossociais, a discriminação de riscos e a coordenação de um tra- 
balho de equipes multiprofissionais, numa perspectiva interdisciplinar, e um dispositivo de gestão para ordenamento dos serviços oferecidos. Vínculo e responsabilização englobariam não só a noção de longitudinalidade, mas a instituição de referências terapêuticas e a responsabilidade do profissional ou da equipe em articular açõese encaminhamentos aos outros recursos disponíveis para resolução dos problemas de saúde da população.

A qualidade da atenção à saúde deve pressupor a referência de um modelo idealizado que leve em conta um conjunto articulado de ações, com efetividade comprovada em determinadas situações de saúde e doença, desenvolvida dentro de uma relação humanizada entre a equipe e os usuários, sendo percebida satisfatoriamente por estes últimos em termos de suas expectativas $^{3}$. Esses conceitos operacionais podem instrumentalizar estudos avaliativos por meio de diferentes estratégias, tais como a observação participante, entrevistas com gestores, profissionais e usuários, pesquisa de documentos e prontuários. Condições traçadoras ${ }^{14} \mathrm{e}$ a "construção" de "linhas de cuidado" ajudam a entender a cadeia produtiva do cuidado e as escol has tecnológicas a serem feitas. Este conjunto de conceitose estratégias metodológicas foi utilizado, com sucesso, em experiências de avaliação e revelaram aspectos importantes dos processos de trabal ho e da integralidade na assistência ${ }^{15}$.

Noções do sistema público de saúde, ampliadas para setor privado:

a universalização da política

nacional de saúde

Ainda que a política nacional de saúde tenha sido proposta com a perspectiva de um sistema de saúde público, universal, não se pode ignorar a expansão do setor suplementar de saúde no Brasil. Este setor abrigava, em junho de 2007, um contingente de 1.679 operadoras de planos privados de saúde responsáveis pelo cuidado à saúde de mais de 46 milhões de vínculos aos planos de saúde (pois um mesmo beneficiário pode possuir mais deum plano), o que representa uma cobertura de $25 \%$ da população brasileira ${ }^{16}$. Justifica-se, assim, que da mesma forma que se deve proceder à avaliação dos serviços públicos de saúde, deve-se procurar igualmente avaliar os serviços privados para regulação do Estado e ampliação da política nacional de saúde a todos os cidadãos, independente de sua escolha no tocante aos serviços para cuidado de sua saúde.
Nesse trabalho, pretendemos analisar aspectos da integralidade na aten ção à saúde nas propostas de avaliação de assistência à saúde de duas instituições, uma nacional e outra internacional, responsáveis pela avaliação do desempenho de outras instituições que oferecem assistência à saúde no âmbito do setor privado: a Agência Nacional de Saúde Suplementar (ANS), órgão regulador do mercado de planos privados de assistência à saúde, no Brasil, e do National Commitee for Quality Assurance (NCQA), organização privada sem fins lucrativos, acreditadora de planos de saúde, nos Estados Unidos.

De forma específica, esses objetivos se traduzem pela: a) descrição das instituições avaliadoras; b) sistematização de suas propostas de avaliação; ec) identificação de aspectos da integralidade nas suas propostas.

\section{Métodos}

Foi efetuada análise documental a partir deinformações obtidas nos sítios eletrônicos das duas instituições, realizada no segundo semestre de 2006. Para descrição das instituiç̧̃̃es, foram destacadas: sua definição, seu tempo de existência e sua missão. Para sistematização das propostas, procurou-se ressaltar aspectos aval iados e mecanismos para obtenção das informações. Por fim, para identificar aspectos da integralidade nas propostas, as informações foram agrupadas segundo atributos pré-definidos numa matriz analítica, conformereferencial teórico explicitado anteriormente. Essa matriz propõe a avaliação da integralidade segundo três dimensões articuladas: a) atributos das práticas de gestão e organização deserviços; b) atributos dosconhecimentos epráticas dos trabalhadores de saúde; e c) atributos das práticas de controle pela sociedade.

A primeira dimensão diz respeito à necessidade de garantir acesso aos diferentes níveis de tecnologia demandados em cada situação para que 0 atendimento prestado tenha efetiva resolutividade. Nesse sentido, procura-se identificar aspectos que indiquem de que forma as instituições articulam seus serviços ( ou searticulam com outros setores) para garantir o acesso da população em qualquer nível decomplexidade, de acordo com a necessidade apreendida a partir do indivíduo, por profissionais de saúde com escuta ampliada, integrados com usuários e equipes.

$\mathrm{Na}$ segunda dimensão, a integralidade pressupõe o estabelecimento de uma relação menos vertical e mais positiva entre os atores (gestores, 
profissionais e usuários) interpretada como um "tratamento digno e respeitoso, com qualidade, acol himento evínculo" equesetraduz numagestão compartilhada do cuidado e dos serviços ${ }^{9}$. Implica uma revisão dos processos de trabal ho e na adoção de novas tecnologias, especialmente as leves e leves-duras, segundo M erhy ${ }^{12}$.

E, por fim, a terceira dimensão relaciona-se com a capacidade das políticas governamentais ordenarem os sistemas de saúde. Pressupõe a superação das práticas sanitárias em sentido restrito ea opção pela construção da li berdadeindividual, da autonomia dos sujeitos e de uma sociedade mais solidária e participativa. Com vistas a esse objetivo, deve-se ter em mente a revisão dos processos de gestão e do planejamento em saúde, bem como a adoção de novas tecnologias de gestão que estimulem e sejam permeáveis à partici pação da sociedadenas decisões de seu interesse e na avaliação de políticas públicas.

\section{Resultados ediscussão}

Descrição das instituições avaliadoras

A Agência Nacional de Saúde Suplementar (ANS) é uma autarquia especial do M inistério da Saúde (M S), criada no ano 200017, com a missão de promover a defesa do interesse público na assistência suplementar à saúde, regular as operadoras setoriais, inclusive quanto às suas relações com prestadores e consumidores e contribuir para o desenvolvimento das ações de saúde no País ${ }^{17}$.

Ao propor uma política de qualificação para o setor privado, a partir de 2004, o órgão regulador pretendeu instituir um novo marco no processo de macro-regulação, com o reconhecimento do campo da saúde suplementar como um local de produção da saúde e a indução a uma transformação dos atores envolvidos: "as operadoras de planos em gestoras de saúde; os prestadores de serviço em produtores de cuidado de saúde; os beneficiários em usuários com consciência sanitária e o próprio órgão regulador"18,19, com vistas a também se aprimorar para corresponder à tarefa de regular um setor com o objetivo de produzir saúde.

O National Committee for Quality Assurance (NCQA) - Comitê Nacional para Garantia de Qualidade - é uma organização americana privada, sem fins lucrativos, fundada em 1990, quetem como missão a melhoria da qualidade do cuidado de saúde $e^{20}$. Iniciou, em 1991, a atividade de acreditação das M anaged Care Organizations (M CO) - organizações quegerenciam autilização eo financiamento de planos privados de saúde - como resposta à necessidade de informação padronizada e objetiva sobre a qualidade do cuidado ${ }^{21}$.

Sistematização das propostas de avaliação (ANS e NCQA)

A proposta de avaliação construída consiste em analisar determinados segmentos de assistência oferecidos pelas diferentes modalidades de operadoras de planos privados de assistência à saúde, em quatro linhas de cuidado no campo da saúde suplementar: (1) materno-neonatal; (2) grupo das neoplasias nas doenças crônicas nãotransmissíveis (DCNT); (3) grupo das doenças cardiovascularesnas doenças crônicas não-transmissíveis (DCNT) (para segmentos médico-hospitalares com ou sem planos odontológicos); e (4) saúde bucal (para o segmento odontológico, em geral). Segundo a ANS, a escolha dessas linhas de cuidado não foi aleatória, mas baseada no perfil de morbi-mortalidade da população usuária dos planos privados de saúde ${ }^{18}$.

No conjunto de indicadores utilizados para avaliar cada uma dessas linhas de cuidado, encontram-se os do âmbito da prevenção, da morbidade e da mortalidade, preconizados pelo MS e definidos após discussão e validação por especialistas de cada uma das áreas contempladas, de forma a compor "retratos" da atenção integral pela articulação das ações de prevenção (diagnóstico precoce) esua influência no adoecimento e morte da população. Esse conjunto de indicadores gera índices de desempenho utilizados no monitoramento das operadoras pela ANS ${ }^{18}$.

Os Índices de Desempenho na Saúde Suplementar (IDSS) são obtidos por meio da análise ponderada de quatro dimensões das operadoras: atenção à saúde (50\%), econômico-financeira (30\%), estrutura e operação ( $10 \%)$ e satisfação do beneficiário (10\%). A pontuação das operadoras é obtida em função de metas a serem alcançadas, usando como referência os parâmetros utilizados pelo setor público. 0 índice é elaborado por indicador, dimensão, conjunto de operadoras por segmentação assistencial (médico-hospitalar e exclusivamente odontológico), classificação e porte, bem como no geral do setor da saúde suplementar.

Além do monitoramento dos indicadores a partir dos sistemas deinformação instituídos pela ANS, o órgão utilizou outras estratégias para monitoramento: uma pesquisa quali-quantitativa junto aos usuários, a fim de avaliar o conhe- 
cimento sobre a agência e a satisfação dos mesmos com as operadoras e com a ANS, e uma investigação multicêntrica pela rede de centros colaboradores da ANS, de natureza qualitativa, por meio de estudos de casos sobre o processo de trabal ho na assistência à saúde e a articulação dos diversos atores, em todas as regiões do país.

A cada etapa de divulgação, são efetuadas discussões sobre o programa com representações de todos os segmentos - operadoras de planos privados de saúde, prestadores de serviços de saúde e beneficiários representados pelos órgãos de defesa do consumidor. 0 desempenho das organizações é disponibilizado aos atores por meio do portal eletrônico na internet.

O National Commitee for Quality Assurance (NCQA) produz e divulga informações sobre planos de saúde para subsidiar o consumidor e o empregador em suas escolhas. As informações são geradas a partir de padrões de qualidade e medidas de desempenho também auxiliares aos diversos atores ligados ao sistema de saúde na identificação de oportunidades de mel horia erealização de mudanças para aperfeiçoamento da qualidade do cuidado oferecido ao paciente ${ }^{21}$. 0 certificado de acreditação do NCQA, obtido de forma voluntária pelas empresas, é reconhecido por mais de trinta estados americanos, pelo Governo Federal e pelo programa M edicare e dispensa os planos acreditados de diversas auditorias estaduais e federais ${ }^{22}$.

Quando solicitado para uma acreditação, uma equipe de peritos treinados na área de saúde é designada pelo NCQA para conduzir uma avaliação rigorosa, dentro e fora das M CO, com mais de sessenta padrões e indicadores. Os avaliadores analisam os sistemas e processos e combinam as informações coletadas no local da avaliação com informações dos registros dos planos de saúde e de pesquisas junto aos consumidores ${ }^{23}$. O plano precisa ter bom desempenho em três dimensões distintas ${ }^{22}$ : estrutura e processos; clínica e satisfação dos usuários.

As estruturas e processos são medidas por padrões de acreditação do órgão e se subdividem em cinco dimensões: acesso, serviço, provedores qualificados, manutenção da saúde, recuperação da doença.

A clínicaéavaliada pelo H ealth Plan Employer Data and Information Set (HEDIS), ferramenta de coleta padronizada de dados, para gerar comparações sobre a qualidade do cuidado e dos serviços oferecidos pelos planos de saúde ${ }^{24}$.

Por fim, a satisfação dos usuários é avaliada pelo Consumer Assessment of Health Plan Survey
(CAHPS), parte do HEDIS, com inúmeros indicadores divididos nas dimensões: efetividade do cuidado, acesso e disponibilidade do cuidado, satisfação com a experiência do cuidado (CAHPS), estabilidade do plano de saúde, uso dos serviços, custo do cuidado, seleção informada sobre opções de tratamento e informações descritivas do plano de saúde.

0 resultado da avaliação de um plano é comparado às médias nacionais dos planos de saúde e utilizado para determinar o resultado geral da acreditação. Um comitênacional avalia esse resultado e atribui ao plano um selo, referenteao nível atingido: excelente, recomendável eacreditado.

0 desempenho das organizações é disponibilizado à população através de portais eletrônicos na Internet.

Identificação de aspectos da integralidade nas propostas de avaliação

do setor privado de saúde

No Quadro 1, procuramos sintetizar a observação das duas experiências relatadas com base na matriz analítica utilizada que considera três dimensões operativas da integralidadee, no Quadro 2, enunciamos as fontes de dados usadas pelas duas organizações.

O bserva-se, no caso brasileiro, umaênfasenos atributos das práticas de gestão e organização de serviços e das práticas de controle pela sociedade. A proposta da ANS toma como ponto de partida a política nacional de saúde e procura regular 0 setor privado com base em informações padronizadas, obtidas num grande número de sistemas de informações desenvolvidos pelo órgão regulador, bem como por meio da articulação destes com os sistemas nacionais de informação, da população brasileira (Quadro 2). 0 programa enfatiza um conjunto de indicadores baseados em quatro dimensões, com pesos diferentes, sendo que a de maior ponderação corresponde à atenção à saúde ( $50 \%$ do peso). N esta dimensão, a categoria central éa linha de cuidado para agravos de prevalência, vulnerabilidadeetranscendência relevante na população brasileira. Entretanto, a avaliação ainda está centrada nos órgãos regulados, as operadoras de planos de saúde, e não nos prestadores de serviços. Os chamados "produtos", designação formal dos planos de saúde no Brasil, não são avaliados.

$\mathrm{N}$ a proposta americana, existe um equilíbrio entre os três conjuntos de atributos das práticas. N essa experiência, são avaliados os aqui (no Brasil) chamados "produtos". Ressalta-se que a ava- 
liação procura detectar a utilização de estratégias semelhantes nos diferentes momentos do pro- cesso de adoecimento (prevenção, redução dos danos e reabilitação). Existe um sistema de in-

Quadro 1. Avaliação da integralidade nas propostas.

\begin{tabular}{|c|c|c|}
\hline $\begin{array}{l}\text { Categorias } \\
\text { de análise }\end{array}$ & ANS & NCQA \\
\hline $\begin{array}{l}\text { Atributos das } \\
\text { práticas de } \\
\text { gestão e } \\
\text { organização de } \\
\text { serviços }\end{array}$ & $\begin{array}{l}\text { Dimensão da atenção à saúde } \\
\text {. Avaliação e monitoramento de } \\
\text { indicadores de prevenção, morbidade e } \\
\text { mortalidade utilizando conceito de } \\
\text { condições traçadoras } \\
\text {. Utilização do conceito de linha de } \\
\text { cuidado: doenças crônicas não } \\
\text { transmissíveis ( diabetes, doenças } \\
\text { cardiovasculares eneoplasias), saúde } \\
\text { materno-infantil; saúde bucal } \\
\text {. Pesquisa multicêntrica em amostra de } \\
\text { operadoras para avaliar processo de } \\
\text { trabalho e microrregulação } \\
\text { Dimensão Estrutura e operação } \\
\text { - Quantidade de beneficiários envolvidos } \\
\text { nas operadoras } \\
\text { - Tempo de adesão ao plano de saúde } \\
\text {. Tempo de realização de determinado } \\
\text { procedimento } \\
\text {. Rede hospitalar da operadora e sua } \\
\text { dinâmica } \\
\text { Dimensão econômico-financeira } \\
\text { - Indicadores de liquidez } \\
\text { - Indicadores de estrutura de capital } \\
\text { - Indicadores de custos } \\
\text { - Indicadores de rentabilidade }\end{array}$ & $\begin{array}{l}\text { Estruturas e processos: } \\
\text {. Acesso } \\
\text {. Serviço } \\
\text {. Provedores qualificados } \\
\text {. M anutenção da saúde } \\
\text {. Recuperação da doença } \\
\text { Clínica } \\
\text {. Health Plan Employer Data and } \\
\text { Information Set (HEDIS): ferramenta } \\
\text { de coleta padronizada de dados, para } \\
\text { gerar comparações sobre a qualidade do } \\
\text { cuidado e dos serviços oferecidos pelos } \\
\text { planos de saúde. }\end{array}$ \\
\hline $\begin{array}{l}\text { A tributos dos } \\
\text { conhecimentos } \\
\text { e práticas dos } \\
\text { trabalhadores } \\
\text { de saúde }\end{array}$ & $\begin{array}{l}\text { Dimensão da atenção à saúde } \\
\text {. D esenvolvimento de programas de } \\
\text { promoção da saúde } \\
\text {. Proposta de gerenciamento do cuidado } \\
\text { ou gerenciamento de casos (case } \\
\text { management) } \\
\text {. Indicadores baseados no uso de } \\
\text { tecnologias duras e leve- duras do } \\
\text { processo de trabalho em saúde } \\
\text {. Linhas de cuidado segundo agravose } \\
\text { grupos de risco } \\
\text {. Proposta de utilização de diretrizes } \\
\text { clínicas pelas operadoras }\end{array}$ & $\begin{array}{l}\text { Clínica } \\
\text {. U tilização de diretrizes clínicas } \\
\text {. Políticas de comunicação sobre direitos } \\
\text { e deveres do usuário e dos médicos } \\
\text {. Promoção da saúde } \\
\text {. Prevenção } \\
\text {. Programa de melhoria contínua } \\
\text { M anutenção da saúde } \\
\text {. Promoção da saúde } \\
\text {. Programa de melhoria contínua } \\
\text {. Utilização de diretrizes clínicas } \\
\text {. M onitoramento da qualidade do } \\
\text { cuidado em condições agudas } \\
\text { Recuperação da doença } \\
\text {. Promoção da saúde } \\
\text { - Gerenciamento da doença: diabetes, } \\
\text { saúde mental, cardiovascular e demais } \\
\text { doenças crônicas } \\
\text {. Políticas de assistência farmacêutica } \\
\text {. Programa de melhoria contínua } \\
\text {. U tilização de diretrizes clínicas }\end{array}$ \\
\hline
\end{tabular}


formações articulado que procura dar conta dos vários aspectos considerados no processo de avaliação (Quadro 2).

As duas propostas procuram aferir a satisfação do beneficiário, sendo que a proposta americana mostra-se mais estruturada e abrangente. Ambas procuram divulgar os resultados de suas avaliações em sítios eletrônicos de forma a permitir que os usuários tenham conhecimento das avaliações.
Refletindo sobre as experiências

Antes de discutirmos as duas experiências, torna-se necessário fazer uma primeira advertência quanto ao cuidado necessário para analisar 0 Quadro 1: uma mesma dimensão avaliada pelas instituições pode estar relacionada a um mesmo conjunto de atributos de práticas.

No que diz respeito aos atributos das práticas de gestão e organização de serviços, as duas

Quadro 1. continuação

\begin{tabular}{|c|c|c|}
\hline $\begin{array}{l}\text { Categorias } \\
\text { de análise }\end{array}$ & ANS & NCQA \\
\hline $\begin{array}{l}\text { Atributos de } \\
\text { práticas de } \\
\text { controle pela } \\
\text { sociedade }\end{array}$ & $\begin{array}{l}\text { Dimensão Satisfação do beneficiário } \\
\text {. Índice de reclamações ao órgão } \\
\text { regulador } \\
\text {. Índice de reclamações aos PROCONs } \\
\text {. Índice de processos transitados em } \\
\text { julgado } \\
\text { Pesquisa nacional quali-quantitativa }\end{array}$ & $\begin{array}{l}\text { Consumer Assessment of Health Plan } \\
\text { Survey (CAHPS) } \\
\text {. Efetividade do cuidado } \\
\text {. Acesso e disponibilidade do cuidado } \\
\text {. Satisfação com a experiência do cuidado } \\
\text { (CAH PS) } \\
\text {. Estabilidade do plano de saúde } \\
\text {. Uso dos serviços } \\
\text {. Custo do cuidado } \\
\text {. Seleção informada sobre opções de } \\
\text { tratamento } \\
\text {. Informações descritivas do plano de saúde }\end{array}$ \\
\hline
\end{tabular}

Quadro 2. Fontes dos dados.

\begin{tabular}{|c|c|c|}
\hline & ANS & NCQA \\
\hline $\begin{array}{l}n \\
0 \\
0 \\
0 \\
0 \\
\text { n } \\
0 \\
y \\
y \\
0 \\
0 \\
0\end{array}$ & $\begin{array}{l}\text { Sistemas de informação da ANS: } \\
\text {. Sistema de Registro de Produtos (RPS) } \\
\text { Sistema de Informações de Beneficiários (SIB) } \\
\text {. Sistema de Informação de Produtos (SIP) } \\
\text { Sistema Integrado de Fiscalização (SI F) } \\
\text {. Informações Periódicas de Operadoras de Planos de Saúde } \\
\text { (DIOPS) } \\
\text {. Informações Periódicas das Seguradoras (FIP) } \\
\text {. Ressarcimento ao SU S } \\
\text {. Cadastro de Operadoras (CADOP) } \\
\text { Sistemas de informação do M S: } \\
\text {. Sistema de Informação de M ortalidade (SIM /SUS) } \\
\text { Sistema de Internação H ospitalar (SIH/SUS) } \\
\text { Estudos multicêntricos de casos } \\
\text { Pesquisa nacional quali-quantitativa }\end{array}$ & $\begin{array}{l}\text { Interactive Survey } \\
\text { System (ISS): } \\
\text { ferramenta online } \\
\text { de avaliação } \\
\text { http://web.ncqa.org/ }\end{array}$ \\
\hline
\end{tabular}


experiências procuram caracterizar a rededeserviços em sua estrutura e operação. No contexto brasileiro, com grandes disparidades na alocação de recursos, énecessário verificar a "real" existência de uma rede ${ }^{25}$. N a abordagem do NCQA, é acrescida uma avaliação qual itativa dos recursos existentes (físicos, humanos, equipamentos, capacitação, certificação, entre outras estruturas). N uma perspectiva futura, pode-se investir nessa avaliação mais ampliada no Brasil com uma ótica e conteúdo mais adequado ao ideário do SUS, tanto no sistema privado quanto público. A existência e o uso de sistemas de informação nas experiências revelam uma preocupação com dados cadastrais, econômico-financeiros, de cobertura e de resultados. Pensamos que sistemas de informação com disponibilidade de dados sobre os usuários em qualquer ponto de atendimento da rede ajudem a construir a integralidade. Facilitando 0 acesso às informações do usuário, evitase a necessidade de recontar sua história em cada atendimento encaminhado e possibilita-se o diálogo entre os profissionais e equipes envolvidos nos atendimentos. Lembramos que a informatização éimportante; porém, não éimprescindível para a circulação de informações na rede de serviços. O uso burocrático da informática pode, inclusive, se tornar uma barreira de acesso à população. Entretanto, a utilização de um grande número de sistemas de informação desarticulados entre si, como ocorre no Brasil, tanto no sistema privado quanto público, implica ineficiência. $\mathrm{Na}$ experiência americana, um sistema integrado com grande parte das informações necessárias é um aspecto positivo.

A adoção de mecanismos de controle de acesso pode ser útil para a regulação, ampliação de acesso e incremento de eqüidade. É importante ter em mente que, no setor suplementar brasileiro, às vezes, esses mecanismos são restritivos ao acesso.

No caso brasileiro, segundo a perspectiva de construção de um sistema, a existência de centrais de regulação dos recursos assistenciais (leitos, vagas em especialidades, exames sofisticados, entre outros) no sistema privado e público pode significar um esforço da gestão em otimizar a utilização e ampliar o acesso pela população beneficiária de planos de saúde e/ ou usuária do sistema público de saúde. Dispositivos degestão colegiada de recursos parecem bons espaços para discussão, negociação e pactuação entregestores e trabal hadores.

Quanto aos atributos dos conhecimentos e práticas dos trabalhadores de saúde, nas duas experiências, há uma ênfase em práticas preventivas em qualquer momento do processo de adoecimento (mais amplo na experiência americana) tanto como forma de ampliar o leque de serviços ofertados quanto como diretriz para as ações desenvolvidas.

Nos dois casos, as práticas em geral são orientadas por diretrizes e protocolos clínicos baseados na "melhor evidência científica" cuja observação denota 0 aspecto racionalizador no sentido da eficácia/eficiência no uso das tecnologias. A existência de programas de capacitação e educação permanente identificada na experiência americana articula-seà idéia do "melhor uso das tecnologias". As tecnologias mencionadas são na sua maioria conhecimentos e práticas articulados voltados para problemas estruturados (doenças), abordando insuficientemente problemas não estruturados ou manifestações de sofrimento inespecífico esingulares dos usuários. O u seja, não se avalia a capacidade das equipes ou serviços no uso de tecnologias de relação (leves) em nenhum dos dois casos. No caso brasileiro, a realização de estudos multicêntricos pelaANSem todas as regiões do país, numa amostra de operadoras de planos de saúde, procura "aproximar" o olhar de quem avalia, a fim de superar esse obstáculo.

Quando pensamos em recursos tecnológicos, tanto mencionamos os do setor saúde, stricto sensu, quanto os de redes sociais de apoio $0^{26,27}$ ou de outros setores (intersetorialidade).

Por fim, como atributos das práticas de controlepela sociedade, nas duas experiências háuma preocupação de "prestação de contas" à sociedade, no sentido de provêla de informações para "melhores escolhas" dos planos de saúde.

No caso brasileiro, algumas operadoras de planos de saúde (como as autogestões) já estruturam consel hos de usuários em seus processos de gestão ${ }^{28}$, mas esse não é um aspecto avaliado pelo órgão regulador. 0 setor público brasileiro tem avançado mais sobre esse aspecto, em vários níveis de gestão, no âmbito dos Conselhos de Saúde. Furtado ${ }^{29}$ já mencionava a importância da avaliação servir ao controle pela sociedade e sugeria comitês de avaliação formados por avaliadores externos, gestores, profissionais e usuários. Ressaltava que a instauração de processos avaliativos que garantissem a inclusão dos vários grupos de interesses nos programas ou serviços avaliados instituiria uma rede de compromissos ecerto controle sobre a efetivação de alterações definidas durante o processo. A geração de informações e a incorporação de outros pon- 
tos de vista no processo avaliativo são importantes, mas não suficientes para produzir mudanças nas práticas institucionais. 0 processo avaliativo deve ser um dispositivo para as negociações entre os vários interesses atuantes e a construção de novos sentidos e significados na atenção à saúde.

A percepção por parte do usuário éum componente importante de processos avaliativos da qualidade do cuidado ${ }^{4,15,30,31}$. Entretanto, a noção de satisfação deusuárioséproblemática, pois carrega componentes subjetivos, às vezes, contraditórios. Nas experiências da ANS e do NCQA, a satisfação do usuário éavaliada de formas diferentes: como volume de reclamações que geraram autuações e penalidades (no caso brasileiro) ou como opiniões sobre o atendimento recebido em situações agudas e crônicas, entre outras (no caso americano). É importante incluir na experiência de avaliação brasileira a percepção da qualidade da assi stência dos usuários (como é feita na experiência americana) e dos trabal hadores na avaliação da integralidade (que não é feita em nenhum dos dois casos).

Possibilitar a vocalização das visões de usuários e trabalhadores de saúde e o diálogo entre elas e a gestão nos parece uma forma de conduzir a integralidade como política no âmbito do setor suplementar de saúde brasileiro.

Deve-se, entretanto, fazer uma outra advertência: tanto em uma experiência analisada quanto na outra, as relações entre usuários, prestadores e operadoras de planos privados são consideradas relações de mercado (menos marcante nas autogestões). Os usuários são percebidos como "consumidores" com direitos assegurados no consumo.

No caso brasileiro, essas relações de consumo do chamado setor suplementar são diferentes das relações estabelecidas no sistema público de saúde, onde estão cidadãos com direitos de cidadania, garantidos pelo Estado. Esta perspectiva reduz a integralidade no setor privado suplementar a um conjunto de serviços discriminados em um contrato e sua garantia éa de cumprimento desse contrato. Antes da regulação do setor no Brasil, existiam contratos com coberturas de serviços diversas, na maioria das vezes parciais, definidas por cálculos atuariais e pelo risco de utilização (a chamada "sinistralidade"), o que limitava os serviços oferecidos à capacidade de pagamento dos usuários. Tal situação gerava e ainda gera, em contratos anteriores à regulação, muitos conflitos no momento de maior necessidade dos usuários, que se vêem cerceados no acesso às tecnologias mais onerosas no momento de uma complicação de sua doença.

A regulação introduzida pela Lei $n$ - 9.656/1998 e a criação da ANS em $2000^{17}$ instituíram coberturas mínimas mais amplas, aumentando a proteção aos usuários em seus contratos. A análise pela ANS das práticas assistenciais das operadoras revelou possibilidades de ampliação de cobertura sem onerar substantivamente os usuários.

Tanto no Brasil quanto nos Estados Unidos, a adoção de model os assistenciais mais cuidadores e a microrregulação têm mostrado resultados na difícil equação de ampliar cobertura de serviços e controlar custos na assistência. Isto nos leva a crer que a discussão da integralidade no setor privado funciona como um tensionador na expansão do acesso a recursos tecnológicos para a solução de problemas da população, na mesma proporção que ocorre no desenvolvimento do sistema público de saúde no Brasil. Cabe lembrar que o setor, tanto no sistema público quanto no sistema privado, também padece dos problemas gerados pelo modelo hegemônico de prestação de serviços de saúde como excessiva medicalização, uso irracional de tecnologias, predomínio das tecnologias duras nas relações com os usuários e até mesmo uma desumanização revestida de ambientes mais confortáveis e promessas de mel hor atendimento ${ }^{32}$. Como conseqüência, algumas questões focadas pelas duas propostas avaliadas são frutos de tensões produzidas pela sociedade em suas reclamações sobre os serviços de saúde. No caso brasileiro, vale ressal tar que, mesmo no sistema público, o fato de 0 acesso ser universal não exclui a necessidade de uma avaliação crítica da utilização dos recursos em qualquer nível do sistema.

\section{A título de conclusão}

Por fim, cabe lembrar que, se pensarmos o processo de mudança de modelo tecnoassistencial pretendido pelo SUS (ANS inclusa) ${ }^{33,34}$, no âmbito público e suplementar, como uma reestruturação produtiva na saúde com transição tecnológica (do predomínio das tecnologias duras ao predomínio das leves) ${ }^{12,13}$, os conceitos eabordagens avaliativas que ajudem a perceber a integralidade atuarão como verificadores da direcionalidade pretendida. Observando as duas experiências à luz das dimensões de práticas de gestão e organização de serviços; conhecimentos e práticas dos trabalhadores de saúde; e práticas de controle pela sociedade foi possível destacar 
alguns conceitos e noções úteis na avaliação da integralidade de redes de serviços de saúde.

A colhimento, vínculo-responsabilização, processo de trabalho, qualidade da aten ção, coordenação do cuidado, percepção da qualidade da atenção pelos usuários e trabalhadores, estrutura, articulação da rede, intersetorialidade, sistema de informação para o cuidado, eqüidade e mecanismo de controle de acesso, gestão participativa, processo avaliativo como dispositivo de controle pela sociedade possibilitam novos ol hares e perspectivas de avaliação dos serviços de saúde, onde a integralidade é vista como uma ação social que resulta da interação democrática entre os atores no cotidiano de suas práticas na oferta do cuidado de saúde nos diferentes níveis de atenção do sistema6.

\section{Colaboradores}

AG da Silva Júnior, M GM Alves, M TM M ascarenhas, VM N Silva eLC de Carval ho participaram da concepção, delineamento eredação do artigo, bem como da análise e interpretação dos dados.

\section{Referências}

1. Giovanella L, Lobato LVC, Carvalho AI, Conill EM, Cunha EM . Sistemas M unicipais de Saúde e a Diretriz da Integralidade da Atenção: Critérios para Avaliação. Saúde em Debate 2002; 26 (60):37-61.

2. Conill EM. Políticas de atenção primária e reformas sanitárias: discutindo a avaliação a partir da análise do Programa Saúde da Família em Florianópolis, Santa Catarina, Brasil, 1994-2000. Cad Saúde Pública 2002; 18(Supl 1):191-202.

3. Conill EM . Avaliação da integralidade: conferindo sentido para os pactos na programação de metas dos sistemas municipais de saúde. Cad Saúde Pública 2004; 20(5):1417-1423.

4. Silva Junior AG, Mascarenhas MT. Avaliação da atenção básica em saúde sob a ótica da integralidade aspectos conceituais e metodológicos. In: Pinheiro R, M attos RA, organizadores. Cuidado: as fronteiras da integralidade. Rio de Janeiro: IM S/UERJ; 2004. p. 241-257.

5. Pinheiro R, Mattos RA, organizadores. Os sentidos da integralidade na atenção e no cuidado à saúde. Rio de Janeiro: IM S/UERJ/ABRASC 0; 2001.

6. Pinheiro $R, M$ attos RA, organizadores. Construção da integralidade: cotidiano, saberes e práticas em saúde. Rio de Janeiro: IM S/UERJ; 2003.

7. Pinheiro R, Mattos RA, organizadores. Cuidado: as fronteiras da integralidade. Rio de Janeiro: IM S/UERJ; 2004.

8. Pinheiro R, Mattos RA, organizadores. Construção social da demanda: direito à saúde; trabalho em equipe; participação e espaços públicos. Rio de Janeiro: CEPESC/UERJ; 2005. 
9. Pinheiro R, Ferla AA, Silva Junior AG. A integralidade na atenção à saúde da população. In: M arins JJN, Rego S, Lampert JB, Corrêa de Araújo JG, organizadores. Educação médica em transformação: instrumentos para a construção de novas realidades. 1ee. São Paulo: Hucitec; 2004. p. 269-284.

10. Ayres JR. Sujeito, intersubjetividade e práticas de saúde. Cien Saude Colet 2001: 6(1):63-72.

11. Ceccim RB. Equipe de Saúde: a perspectiva entredisciplinar na produção dos atos terapêuticos. In: Pinheiro R, Mattos RA, organizadores. Cuidado: as fronteiras da integralidade. Rio de Janeiro: IM S/UERJ; 2004. p. 259-278.

12. M erhy EE. Saúde: a cartografia do trabalho vivo. São Paulo: Hucitec; 2002.

13. Merhy EE. Em busca de ferramentas analisadoras das tecnologias em saúde: a informação e o dia-adia de um serviço, interrogando e gerindo o trabaIho em saúde. In: M erhy $E E$, Onocko R, organizadores. Agir em saúde. Um desafio para o público. São Paulo: Hucitec; 1997. p. 113-150.

14. Kessner DM, Kalk CE, Singer J. Evaluación de la calidad de la salud por el método de los trazadores. In: Organização Pan-americana de Saúde. Investigaciones sobre servicios de la Salud. Brasília: OPAS; 1992. p. 555-63.

15. Mascarenhas MT. Implementação da atenção básica em saúde no M unicípio de N iterói - RJ. Estudo de caso em unidade básica de saúde tradicional e módulo do Programa M édico de Família [tese ]. Rio de Janeiro (RJ): Escola Nacional de Saúde Pública, Fundação Oswaldo Cruz; 2003.
16. Brasil. M inistério da Saúde. Agência Nacional de Saúde Suplementar. Caderno de Informação da Saúde Suplementar: beneficiários, operadoras e planos. Rio de Janeiro: AN S; 2007. [acessado 2007 Out 18]. Disponível em: http://www.ans.gov.br/portal/site/ informacoesss/informacoesss.asp

17. Lei 9.961 de 28 de janeiro de 2000. Cria a Agência Nacional de Saúde Suplementar - ANS e dá outras providências. [acessado 2007 Out 18]. Disponível em: http://www.ans.gov.br/portal/site/legislacao/ legislacao_integra. asp?id_original $=8$

18. Agência Nacional de Saúde Suplementar. Duas faces da mesma moeda: microrregulação e modelos assistenciais na saúde suplementar. Série A. N ormas e M anuais Técnicos. Rio de Janeiro; ANS; 2005.

19. Agência Nacional de Saúde Suplementar. Texto completo da qualificação da saúde suplementar. 2005. [acessado 2006 Jun 5]. Disponível em: http://www.ans. gov.br/portal/site/_qualificacao/pdf/texto_base.pdf

20. National Committee for Quality Assurance. About NCQA. 2006. [acessado 2006 Jun 10]. Disponível em: http://web.ncqa.org

21. National Committee for Quality Assurance. Overview. 2006. [acessado 2006 Jun 10]. Disponível em: http://web.ncqa.org/tabid/67/D efault.aspx

22. National Committee for Quality Assurance. M anaged Care Organizations/Prefered Provider Organizations. Accreditation Programs. 2006. [acessado 2006 Jun 10]. Disponível em: http://web.ncqa.org/tabid/ 99/D efault.aspx 
23. National Committee for Quality Assurance. NCQA Advertising Guidelines for Accreditation/Certification. 2006. [acessado 2006 Jun 10]. Disponível em: http:/ /www.ncqa.org/M arketing/M CO.pdf

24. National Committee for Quality Assurance. NCQA $H$ ealthcare Effectiveness Data and Information Set (HEDIS) \& Quality M easurement. [acessado 2006 Jun 10]. Disponível em: http://web.ncqa.org/tabid/ 59/D efault.aspx

25. Hartz ZM A, Contandrioupoulos AP. Integralidade da atenção e integração de serviços de saúde: desafios para avaliar a implantação de um "sistema sem muros". Cad Saúde Pública 2004; 20 (Supl 2):331-336

26. Valla VV, Guimarães M B, Lacerda A. Religiosidade, apoio social e cuidado integral à saúde: uma proposta de investigação voltada para as classes populares. In: Pinheiro R, M attos RA, organizadores. Cuidado: as fronteiras da integralidade. Rio de Janeiro: IM S/UERJ; 2004. p. 103-17.

27. Lacerda A, Valla VV. As práticas terapêuticas de cuidado integral à saúde como proposta para aliviar o sofrimento. In: Pinheiro R, M attos RA, organizadores. Cuidado: as fronteiras da integralidade. Rio de Janeiro: IM S/UERJ; 2004. p. 91-102.

28. Silva Junior AG, Haddad R, M elin AR. Conselho de usuários em empresa de autogestão em saúde. In: Anais do Congresso Brasileiro de Ciências Sociais em Saúde. São Paulo: ABRASCO; 1999.

29. Furtado JP. Um método construtivista para a avaliação em saúde. Cien Saude Colet 2001; 6 (1):165-181.
30. Starfield B, Cassady C, Nanda J, Forrest CB, Berk R. Consumer experiences and provider perceptions of the quality of primary care: implications for managed care. J Fam Pract 1998; 46:216-25.

31. Starfield B. Atenção primária: equilíbrio entre necessidades de saúde, serviços e tecnologia. Brasília: Ministério da Saúde; 2002.

32. Silva Junior AG, M erhy EE, Carvalho LC. Refletindo sobre 0 ato de cuidar da saúde. In: Pinheiro R, $M$ attos RA, organizadores. Construção da integralidade: cotidiano, saberes e práticas em saúde. Rio de Janeiro: IM S/UERJ; 2003. p. 89-112.

33. Silva Junior AG. M odelos tecnoassistenciais em saúde: 0 debate no campo da saúde coletiva. São Paulo: Hucitec; 1998.

34. Silva Junior AG, Alves CA, Alves M GM. Entre tramas e redes: cuidado e integralidade. In: Pinheiro R, M attos RA, organizadores. Construção social da demanda: direito à saúde; trabalho em equipe; participação e espaços públicos. Rio de Janeiro: CEPESC/ UERJ; 2005. p. 77-89.

Artigo apresentado em 20/08/2007

Aprovado em 10/10/2007

Versão final apresentada em 30/11/2007 